\title{
High-field magnetization of rare-earth ions in scandium
}

\author{
Roeland, L. W.; Touborg, P.
}

Published in:

Physical Review B

Link to article, DOI:

10.1103/PhysRevB.17.2324

Publication date:

1978

Document Version

Publisher's PDF, also known as Version of record

Link back to DTU Orbit

Citation (APA):

Roeland, L. W., \& Touborg, P. (1978). High-field magnetization of rare-earth ions in scandium. Physical Review B, 17(5), 2324-2327. https://doi.org/10.1103/PhysRevB.17.2324

\section{General rights}

Copyright and moral rights for the publications made accessible in the public portal are retained by the authors and/or other copyright owners and it is a condition of accessing publications that users recognise and abide by the legal requirements associated with these rights.

- Users may download and print one copy of any publication from the public portal for the purpose of private study or research.

- You may not further distribute the material or use it for any profit-making activity or commercial gain

- You may freely distribute the URL identifying the publication in the public portal

If you believe that this document breaches copyright please contact us providing details, and we will remove access to the work immediately and investigate your claim 


\title{
High-field magnetization of rare-earth ions in scandium
}

\author{
L. W. Roeland \\ Natuurkundig Laboratorium, University of Amsterdam, The Netherlands \\ P. Touborg \\ Physics Department, * University of Odense, 5000 Odense, Denmark \\ and Department of Electrophysics, Technical University, Lyngby, Denmark
}

(Received 26 September 1977)

\begin{abstract}
The magnetic moments of $\mathrm{Tb}, \mathrm{Dy}$, or $\mathrm{Er}$ ions in dilute $\mathrm{Sc}$ single-crystal alloys have been measured in fields up to $280 \times 10^{5} \mathrm{~A} / \mathrm{m}(350 \mathrm{kOe})$. The Zeeman energies in this high field are comparable to the total crystal-field splittings. This gives rise to characteristic features in the magnetization curves. The crystal-field parameters obtained previously from experiments in low fields and the Zeeman interaction give a satisfactory quantitative acount of the experimental results.
\end{abstract}

Crystal-field parameters for each of the heavy rare-earth ions $\mathrm{Tb}, \mathrm{Dy}, \mathrm{Ho}, \mathrm{Er}$, and $\mathrm{Tm}$ in each of the nonmagnetic hcp-metal hosts Sc, Y, or Lu have been published earlier. ${ }^{1-3}$ The parameters were deduced from measurements of the initial paramagnetic susceptibility of the rare earth solutes as a function of temperature and crystallographic orientation. The initial susceptibilities were generally measured in a field of $1.35 \times 10^{5}$ $\mathrm{A} / \mathrm{m}$. Magnetization measurements in high fields offer a powerful means of checking these crystalfield parameters. By high fields is meant that the Zeeman energies are comparable to the overall crystal-field splittings, so that information is obtained on the energies and wave functions of the complete multiplet. In the present paper we report on magnetization measurements in fields up to $280 \times 10^{5} \mathrm{~A} / \mathrm{m}$ on the single-crystal alloys Sc, 0.561 -at. \% Tb; Sc, 0.562-at. \% Dy; and Sc, 0.528at. \% Er. The crystal-field splittings for rareearth solutes in a Sc host are smaller than, or approximately equal in magnitude, to the corresponding splittings in $\mathrm{Y}$ and Lu hosts. The effects of the Zeeman interaction on the crystal-field levels are therefore most pronounced for Sc alloys. High-field magnetization measurements on alloys of $\mathrm{Tb}, \mathrm{Dy}$, or $\mathrm{Er}$ in $\mathrm{Y}$ hosts have been reported earlier. ${ }^{4}$

The experiments were performed at the highfield magnet facility at the University of Amsterdam. The magnetic moments of the rare earth solutes were determined from separate measurements of the magnetic moments of the alloy samples and of a pure Sc sample. The uncertainties inherent in the magnetic moment measurements have been discussed by Roeland et $a l^{5,6}$ The absolute uncertainty of the magnetic moment of the rare-earth solute is approximately $\pm 0.5 \mu_{B}$ /atom at the highest field for the samples of the present paper. The experimental magnetic moments at
$4.2 \mathrm{~K}$ in the magnetically hard and easy direction of $\mathrm{Tb}$, Dy, or Er solutes in Sc are shown in Fig. 1.

The Hamiltonian used for calculating the magnetic moments comprises the crystal-field terms and the Zeeman term

$$
\hat{\mathfrak{C}}=B_{20} \hat{O}_{20}+B_{40} \hat{O}_{40}+B_{60} \hat{O}_{60}+B_{66} \hat{O}_{66}+g_{J} \mu_{B} \hat{J} \cdot \overrightarrow{\mathrm{H}} .
$$

The $B_{l m}$ 's are the crystal-field parameters deduced in Refs. 1-3 and quoted in Table $I$. The $\hat{O}_{l m}$ 's are the Stevens operator, ${ }^{7} g_{J}$ is the Lande factor, $\mu_{B}$ is the Bohr magneton, $\hat{J}$ is the angular-momentum operator, and $\overrightarrow{\mathrm{H}}$ is the magnetic field. Exchange interactions have been neglected. In Ref. 3 this is shown to be justified for the Er and Dy alloys, ${ }^{3}$ whilst for the Tb alloys a small molecular-field parameter gives a better fit. The magnetic moment at temperature $T$ in the direction $\nu$ is given by

$M_{\nu}=\sum_{i}\left\langle i\left|-g_{J} \mu_{B} J_{\nu}\right| i\right\rangle e^{-E_{i} / k T} / \sum_{i} e^{-E_{i} / k T}$.

The energy levels $E_{i}$ and eigenfunctions $|i\rangle$ are calculated by diagonalizing the Hamiltonian (1) within the basis $\left|J, J_{z}\right\rangle$ of the lowest lying multiplet of the $L, S$ ground term. Higher-lying multiplets are found to have negligible effects on the magnetic moment. Using the crystal-field parameters (Table I) deduced for each alloy separately the fully drawn magnetization curves in Fig. 1 are calculated. The experimental data are reproduced within the stated accuracy except for $\mathrm{Tb}$ at lower fields in the $c$ direction. For the $\mathrm{Tb}$ alloy studied ordering phenomena have been observed ${ }^{3}$ at $4.2 \mathrm{~K}$ and discrepancies in the $c$ direction between the uncorrected experimental data and theoretical crystal-field predictions are therefore to be expected. Even so the fields at which the characteristic changes of shapes occur are reproduced. 

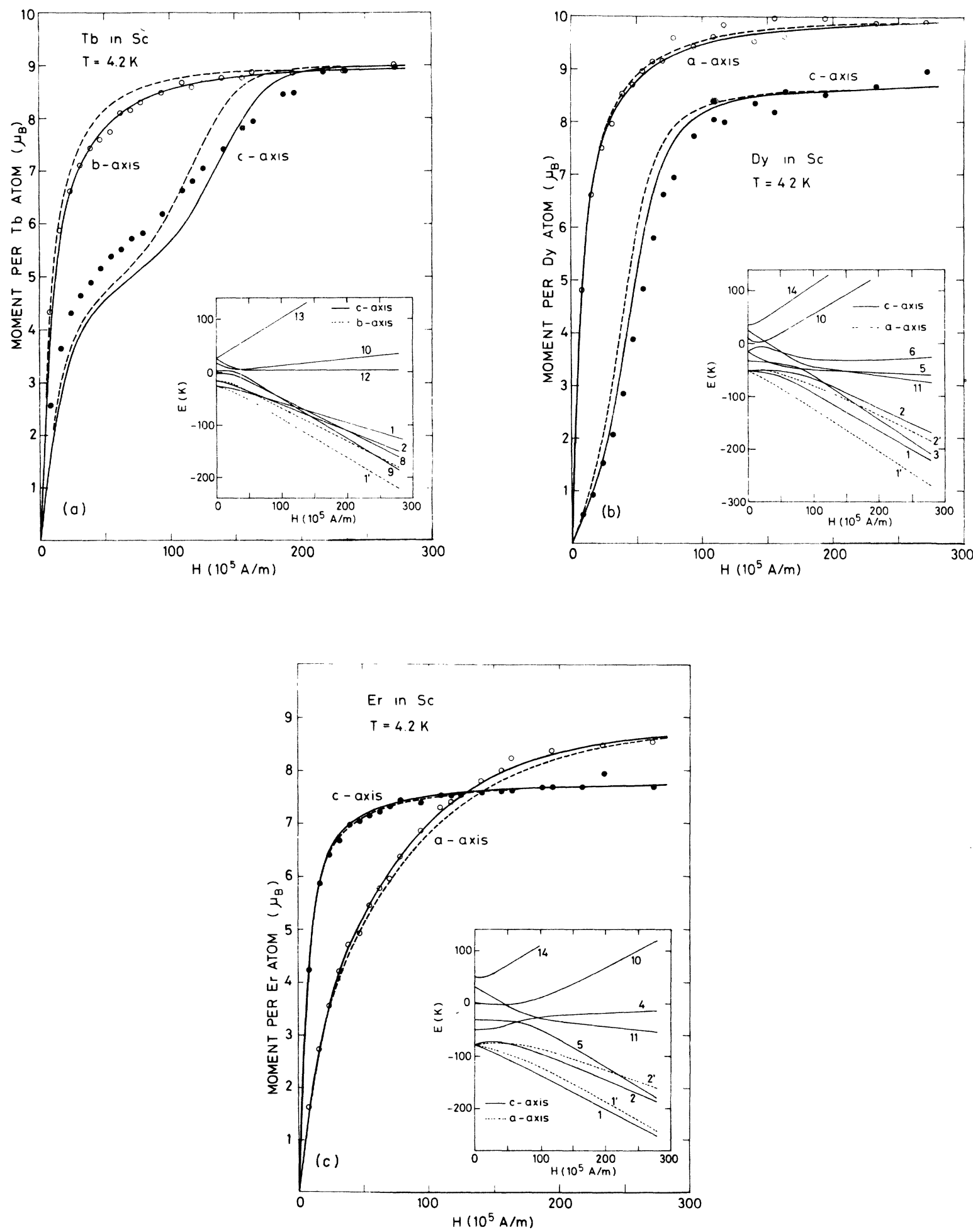

FIG. 1. Magnetization vs field for (a) Sc, 0.561-at.\% Tb; (b) Sc, 0.562-at.\% Dy; and (c) Sc, 0.528-at.\% Er. The energy levels are plotted as a function of the field in the insets. The numbers on the curves refer to the level numbers in Table II. The dashed magnetization curves are calculated using the average crystal-field parameters Table I. All other curves in these figures are calculated using the individual parameter shown in Table I. 
TABLE I. Crystal-field parameters for rare-earth solutes in Sc divided by the Stevens factor (Ref. 7). The average values are calculated using parameters (Ref. 1) for all solutes $\mathrm{Tb}, \mathrm{Dy}, \mathrm{Ho}, \mathrm{Er}$, and $\mathrm{Tm}$. For all alloys $B_{66}$ has a value close to $\frac{\pi}{8} \times B_{60}$ as shown in Ref. 1 .

\begin{tabular}{llcl}
\hline \hline & $B_{20} / \alpha$ & $B_{40} / \beta$ & $B_{60} / \gamma$ \\
\hline Tb in Sc & $-31.0 \pm 4$ & $13.4 \pm 5$ & $24.4_{-5}^{+2}$ \\
Dy in Sc & $-29.9 \pm 3$ & $14.6 \pm 4$ & $20.9 \pm 3$ \\
Er in Sc & $-29.0 \pm 3$ & $8.2_{+5}^{-2}$ & $18.1 \pm 2$ \\
Average values & $-29.5 \pm 0.9$ & $9.9 \pm 1.9$ & $19.8 \pm 1.5$ \\
\hline \hline
\end{tabular}

The dashed magnetization curves, which are calculated using the average crystal-field parameters in Table I reproduce the experimental data less satisfactorily.

The insets in Fig. 1 and Table II give energy levels and wave functions for the rare-earth solutes. The magnetic moment is determined by the slope of the lowest populated levels according to $\left\langle i\left|-g_{J} \mu_{B} J_{\nu}\right| i\right\rangle=-\partial E_{\imath} / \partial H_{\nu}$ in formula (2). The origin of the features in the high field magnetization is apparent from the insets and Table II. The

TABLE II. Crystal-field levels for rare-earth solutes in Sc. The level numbers refer to the levels plotted in Fig. 1. The wave functions are given in the $\left|J, J_{c}\right\rangle=\left|J_{c}\right\rangle$ representation. The last column quotes approximate wave functions at $H=280 \times 10^{5} \mathrm{~A} / \mathrm{m}$ in the $c$ direction. Approximate wave functions at this field for the levels $1^{\prime}$ and $2^{\prime}$ plotted in Fig. 1 are $\left|J, J_{b}\right\rangle=|6,-6\rangle$ and $|6,-5\rangle$ for $\mathrm{Tb} ;\left|J, J_{a}\right\rangle=\left|\frac{15}{2},-\frac{15}{2}\right\rangle$ and $\left|\frac{15}{2},-\frac{13}{2}\right\rangle$ for $\mathrm{Dy}$; and $\left|J, J_{a}\right\rangle=\left|\frac{15}{2},-\frac{15}{2}\right\rangle$ and $\left|\frac{15}{2},-\frac{9}{2}\right\rangle$ for $\mathrm{Er}$.

\begin{tabular}{|c|c|c|c|}
\hline \multicolumn{2}{|c|}{$\begin{array}{c}\text { Level no. } \\
\text { energy (K) at } H=0\end{array}$} & Wave functions at $H=0$ & Wave functions at $H_{c}=35 T$ \\
\hline \multicolumn{4}{|c|}{$\mathrm{Tb}$ in $\mathrm{Sc}$} \\
\hline 1 & -26.8 & $0.71|-3\rangle-0.71|3\rangle$ & $|-3\rangle$ \\
\hline 2,3 & -18.5 & $0.61|\mp 4\rangle-0.79| \pm 2\rangle$ & $|-4\rangle,-|-2\rangle$ \\
\hline 4 & -10.9 & $0.71|-3\rangle+0.71|3\rangle$ & $|3\rangle$ \\
\hline 5,6 & -3.5 & $0.79|\mp 4\rangle+0.61| \pm 2\rangle$ & $|2\rangle,|4\rangle$ \\
\hline 7,8 & -3.4 & $0.96|\mp 1\rangle-0.28| \pm 5\rangle$ & $|-1\rangle,-|-5\rangle$ \\
\hline 9 & 2.6 & $0.12|-6\rangle-0.98|0\rangle+0.12|6\rangle$ & $|-6\rangle$ \\
\hline 10,11 & 16.8 & $0.96|\mp 5\rangle+0.29| \pm 1\rangle$ & $|1\rangle,|5\rangle$ \\
\hline 12 & 25.8 & $-0.71|-6\rangle+0.71|6\rangle$ & $|0\rangle$ \\
\hline 13 & 26.8 & $0.70|-6\rangle+0.17|0\rangle+0.70|6\rangle$ & $|6\rangle$ \\
\hline \multicolumn{4}{|c|}{ Dy in Sc } \\
\hline 1,2 & -52.3 & $0.29\left|\mp \frac{13}{2}\right\rangle+0.92\left|\mp \frac{1}{2}\right\rangle+0.26\left| \pm \frac{11}{2}\right\rangle$ & $\left|-\frac{13}{2}\right\rangle,\left|-\frac{11}{2}\right\rangle$ \\
\hline 3,4 & -32.7 & $0.09\left|\mp \frac{15}{2}\right\rangle+0.94\left|\mp \frac{3}{2}\right\rangle+0.33\left| \pm \frac{9}{2}\right\rangle$ & $\left|-\frac{15}{2}\right\rangle,\left|-\frac{9}{2}\right\rangle$ \\
\hline 5,6 & -15.5 & $-0.95\left|\mp \frac{13}{2}\right\rangle+0.25\left|\mp \frac{1}{2}\right\rangle+0.20\left| \pm \frac{11}{2}\right\rangle$ & $\left|-\frac{1}{2}\right\rangle,\left|\frac{1}{2}\right\rangle$ \\
\hline 7,8 & -10.9 & $0.53\left|\mp \frac{7}{2}\right\rangle+0.85\left| \pm \frac{5}{2}\right\rangle$ & $\left|-\frac{7}{2}\right\rangle,\left|-\frac{5}{2}\right\rangle$ \\
\hline 9,10 & 10.2 & $0.12\left|\mp \frac{13}{2}\right\rangle-0.31\left|\mp \frac{1}{2}\right\rangle+0.94\left| \pm \frac{11}{2}\right\rangle$ & $\left|\frac{11}{2}\right\rangle,\left|\frac{13}{2}\right\rangle$ \\
\hline 11,12 & 25.4 & $-0.98\left|\mp \frac{15}{2}\right\rangle+0.02\left|\mp \frac{3}{2}\right\rangle+0.20\left| \pm \frac{9}{2}\right\rangle$ & $\left|-\frac{3}{2}\right\rangle,\left|\frac{3}{2}\right\rangle$ \\
\hline 13,14 & 35.0 & $0.18\left|\mp \frac{15}{2}\right\rangle-0.34\left|\mp \frac{3}{2}\right\rangle+0.92\left| \pm \frac{9}{2}\right\rangle$ & $\left|\frac{9}{2}\right\rangle,\left|\frac{15}{2}\right\rangle$ \\
\hline 15,16 & 40.7 & $-0.85\left|\mp \frac{7}{2}\right\rangle+0.53\left| \pm \frac{5}{2}\right\rangle$ & $\left|\frac{5}{2}\right\rangle,-\left|\frac{7}{2}\right\rangle$ \\
\hline $\mathrm{Er}$ in $\mathrm{Sc}$ & -78.4 & $0.83\left|\mp \frac{13}{2}\right\rangle-0.48\left|\mp \frac{1}{2}\right\rangle+0.27\left| \pm \frac{11}{2}\right\rangle$ & $\left|-\frac{13}{2}\right\rangle,\left|-\frac{11}{2}\right\rangle$ \\
\hline 3,4 & -48.7 & $0.52\left|\mp \frac{13}{2}\right\rangle+0.52\left|\mp \frac{1}{2}\right\rangle-0.68\left| \pm \frac{11}{2}\right\rangle$ & $\left|-\frac{1}{2}\right\rangle,\left|\frac{1}{2}\right\rangle$ \\
\hline 5,6 & -30.4 & $0.12\left|\mp \frac{15}{2}\right\rangle-0.84\left|\mp \frac{3}{2}\right\rangle+0.53\left| \pm \frac{9}{2}\right\rangle$ & $\left|-\frac{15}{2}\right\rangle,\left|-\frac{9}{2}\right\rangle$ \\
\hline 7,8 & -5.3 & $0.63\left|\mp \frac{7}{2}\right\rangle-0.78\left| \pm \frac{5}{2}\right\rangle$ & $\left|-\frac{7}{2}\right\rangle,-\left|-\frac{5}{2}\right\rangle$ \\
\hline 9,10 & 2.4 & $0.19\left|\mp \frac{13}{2}\right\rangle+0.71\left|\mp \frac{1}{2}\right\rangle+0.68\left| \pm \frac{11}{2}\right\rangle$ & $\left|\frac{11}{2}\right\rangle,\left|\frac{13}{2}\right\rangle$ \\
\hline 11,12 & 31.6 & $-0.96\left|\mp \frac{15}{2}\right\rangle+0.40\left|\mp \frac{3}{2}\right\rangle+0.29\left| \pm \frac{9}{2}\right\rangle$ & $\left|-\frac{3}{2}\right\rangle,\left|\frac{3}{2}\right\rangle$ \\
\hline 13,14 & 51.3 & $0.26\left|\mp \frac{15}{2}\right\rangle+0.55\left|\mp \frac{3}{2}\right\rangle+0.80\left| \pm \frac{9}{2}\right\rangle$ & $\left|\frac{9}{2}\right\rangle,\left|\frac{15}{2}\right\rangle$ \\
\hline 15,16 & 77.6 & $0.78\left|\mp \frac{7}{2}\right\rangle+0.63\left|\mp \frac{5}{2}\right\rangle$ & $\left|\frac{5}{2}\right\rangle,\left|\frac{7}{2}\right\rangle$ \\
\hline
\end{tabular}


characteristic changes of slope for increasing fields in the $c$-axis magnetization of $\mathrm{Tb}$ result from the crossing through the ground state 1 of the states 2 and 9. For increasing fields along the $c$ axis in Dy the level 5 moves down and mixes with the ground state 1 changing this from mainly $\left|J, J_{c}\right\rangle=\left|\frac{15}{2},-\frac{1}{2}\right\rangle$ to mainly $\left|\frac{15}{2},-\frac{13}{2}\right\rangle$. This accounts. for the sharp increase in the $c$-axis magnetization.

For fields in the $c$ direction the ground-state level 1 in $\mathrm{Er}$ is mainly $\left|J, J_{c}\right\rangle=\left|\frac{15}{2},-\frac{13}{2}\right\rangle$. For increasing fields in the $a$ direction the ground state $1^{\prime}$ changes continuously towards $\left|J, J_{a}\right\rangle=\left|\frac{15}{2},-\frac{15}{2}\right\rangle$. This accounts for the crossing of the $a$-axis and $c$-axis magnetization curves for Er.

The high-field magnetization for the Sc alloys are similar to those observed earlier for $\mathrm{Y}$ alloys. ${ }^{4,1}$ The characteristic features occur at lower fields for Sc-Tb and Sc-Dy than for Y-Tb and
Y-Dy. This reflects the smaller value of $B_{20}$ in Sc alloys.

The crystal-field parameters $B_{l m} / \alpha_{1}$ deduced for the individual solutes give a somewhat better fit to the high-field magnetization data for the Sc alloys than do the average values for all rareearth solutes in Sc. This indicates that there is a small but significant dependence of the effective crystal field on the rare-earth ion on which it acts.

\section{ACKNOWLEDGMENTS}

The authors acknowledge fruitful discussions with J. Hog, V. Frank, A. R. Mackintosh, and F. A. Muller. The present research has benefitted from financial support through the NATO Research Grant Program.
*Present address.

${ }^{1}$ P. Touborg, Phys. Rev. B 16, 1201 (1977).

${ }^{2}$ O. Rathmann and P. Touborg, Phys. Rev. B 16, 1212 (1977).

${ }^{3} \mathrm{~J} . \mathrm{H} \phi g$ and P. Touborg, Phys. Rev. B 14, 1209 (1976).

${ }^{4}$ P. Touborg, J. Høg, G. J. Cock, and L. W. Roeland, Phys. Rev. B 10, 2952 (1974). (Note that corrections for magnetic ordering performed in Ref. 1 have changed significantly the crystal-field parameter for Dy in $Y$. The correct theoretical high-field magne- tization for Dy in $\mathrm{Y}$ showing a much better agreement with experiment is shown in Fig. 7 in Ref. 1.)

${ }^{5}$ L. W. Roeland, F. A. Muller, and R. Gersdorf, Coll. Int. Cent. Natl. Rech. Sci. 166, 175 (1967).

${ }^{6}$ L. W. Roeland, G. J. Cock, F. A. Muller, and D. Schoenberg, Physica (Utr.) B 79, 95 (1975).

${ }^{7}$ A. Abragam and B. Bleaney, Electron Paramagnetic Resonance of Transition Ions (Clarendon, Oxford, 1970). 